\title{
RESEARCH HIGHLIGHT Remote light-activation of native Orai channels
}

\author{
Sarah A. Kazzaz ${ }^{1}$, James H. Baraniak Jr${ }^{1}$, Yandong Zhou ${ }^{1}$ and Donald L. Gill (D) \\ Cell Research (2021) 31:727-729; https://doi.org/10.1038/s41422-021-00484-y
}

\begin{abstract}
Optogenetic control of the $\mathrm{Ca}^{2+}$ signaling machinery has been a powerful approach but requires complex cell manipulation to introduce modified genes. A newly developed non-invasive, ultrafast laser technique allows for the remote activation of native Orai $1 \mathrm{Ca}^{2+}$ channels, without the need for gene introduction, protein expression, or exogenous reagents.
\end{abstract}

$\mathrm{Ca}^{2+}$ is an essential controller of innumerable cellular processes including transcription, cell growth, apoptosis, immune responses, and neuronal excitation. Intracellular $\mathrm{Ca}^{2+}$ signals and stores are coordinated through "store-operated" $\mathrm{Ca}^{2+}$ entry (SOCE), controlled by the STIM/Orai system. ${ }^{1}$ Upon sensing lowered endoplasmic reticulum (ER) $\mathrm{Ca}^{2+}$, STIM unfolds and extends from the ER to tether to and activate plasma membrane (PM) Orai channels within ER-PM junctions, mediating $\mathrm{Ca}^{2+}$ entry signals. Aberrant STIM/Orai functioning is implicated in a large number of immunological, muscular, and neurological disorders. Hence, there is much interest in methods to non-invasively manipulate and understand the function of SOCE in different cell types. ${ }^{1}$

Optogenetics, in which cells are genetically engineered to express light-sensitive proteins, offers a promising approach to remotely activate SOCE. For example, it has been possible to engineer photosensitive domains within the cytosolic portion of STIM1, and use them to remotely light-activate $\mathrm{Ca}^{2+}$ entry signals through Orai channels. ${ }^{2,3}$ These optogenetic tools, coined OptoSTIM1 and OptoCRAC, demonstrate therapeutic potential, for example, to enhance targeting of antigen-specific immune responses in cancer cell immunotherapy, and augment stem cell colonies to enhance neural development. ${ }^{2,3}$ Recently, He et al. ${ }^{4}$ engineered a light-oxygen-voltage domain directly into the intracellular loop of a constitutively active form of Orai1, to form a powerful light-operated $\mathrm{Ca}^{2+}$ channel (LOCa). This was used to precisely generate $\mathrm{Ca}^{2+}$ signals to remotely control transcription and cell growth in tissues.

The obvious drawback with such technology is the need to deliver optogenetic variants into cells and tissues to render them sensitive to modification by light. Recent advances reveal that penetrating near-infrared femtosecond lasers can be precisely targeted to photo-activate cells, generating low-energy photons that can modify function while causing minimal cellular damage. Indeed, dual-photon lasers allow delivery of high photon density with great spatiotemporal accuracy. Earlier studies revealed that femtosecond lasers could directly activate $\mathrm{Ca}^{2+}$ signals in cells $s^{5,6}$ although the target(s) and mechanism for such activation were unclear. Now, in a pioneering study from Cheng et al. ${ }^{7}$ femtosecond laser technology is revealed to target native Orai1 channels, providing a potentially crucial means to non-invasively photoinduce $\mathrm{Ca}^{2+}$ signals in cells without any cellular manipulation or altered expression of proteins.
This novel technology (named femtoSOC) is both exciting and enigmatic. Using a standard multiphoton confocal microscope, focusing low-power femtosecond laser pulses on a $2 \times 2 \mu \mathrm{m}^{2}$ area of the PM induces rapid $\mathrm{Ca}^{2+}$ entry within the targeted area. This effect is shown in a variety of different cell types. Remarkably, cells in which all three Orai channels were genetically knocked out by CRISPR, have no femtoSOC $\mathrm{Ca}^{2+}$ entry; but expression of Orai1 restores the $\mathrm{Ca}^{2+}$ entry. Importantly, knocking out STIM1 has no effect on this femtoSOC. Indeed, the femtosecond laser application has no effect on ER-stored $\mathrm{Ca}^{2+}$ or on the translocation of STIM1. Thus, the mechanism of Orai channel activation is independent of the "store-operated" STIM-dependent coupling across ER-PM junctions that normally activates Orai channels. ${ }^{1}$ Moreover, using a range of Orai channel inhibitors, the femtoSOC $\mathrm{Ca}^{2+}$ entry appears to have the same pharmacological profile as native SOCE. Although Orai 1 is the predominant Orai channel subtype in many cell types, the work did not ascertain whether femtoSOC activates the Orai2 or Orai3 subtypes when expressed in isolation.

Understanding the mechanistic basis for this extraordinary and specific action of femtosecond laser-induced Orai channel activation presents some uncertainty. Cheng et al. describe "aggregation" of Orai channels within the laser-scanned areas, which they interpret as being mediated by assembly of Orai1 channel subunits into their now-established hexameric functional configuration. ${ }^{1}$ They support this conclusion with laserenhanced FRET measurements between CFP- and YFP-tagged Orai1. However, the widespread existence of PM Orai1 dimers is unlikely. Like many channels, Orai 1 channels exist mostly in their functional multi-subunit state, and it does not appear that STIM proteins activate Orai1 channels by inducing hexameric assembly. The observed "aggregation" of Orai1 in laser-targeted areas looks to be clustering and trapping of pre-assembled channels. This clustering seems to occur over a period of several minutes, and appears to be slower than the onset of $\mathrm{Ca}^{2+}$ entry which occurs in seconds. Thus, it is possible that channel activation and translocation are separate events (see Fig. 1). Alternatively, femtoSOC-induced micro-assembly of channel clusters (prior to the later macro-aggregation), may be an important part of the mechanism of channel activation. Indeed, STIM-induced Orai channel clustering greatly enhances store-dependent Orai channel activation.

What of the molecular basis for the light-induced Orai1 channel activation? The dual photon-induced light spectrum of activation suggested the isoalloxazine footprint of flavins. ${ }^{7}$ Indeed, photoexcitation of the recently engineered OptoCRAC molecule involves covalent binding of FMN to a cysteine in the photosensitive region. ${ }^{2}$ Cheng et al. ${ }^{7}$ present biochemical studies supporting the proposal that photoexcitation of cytosolic flavins initiates Orai1 

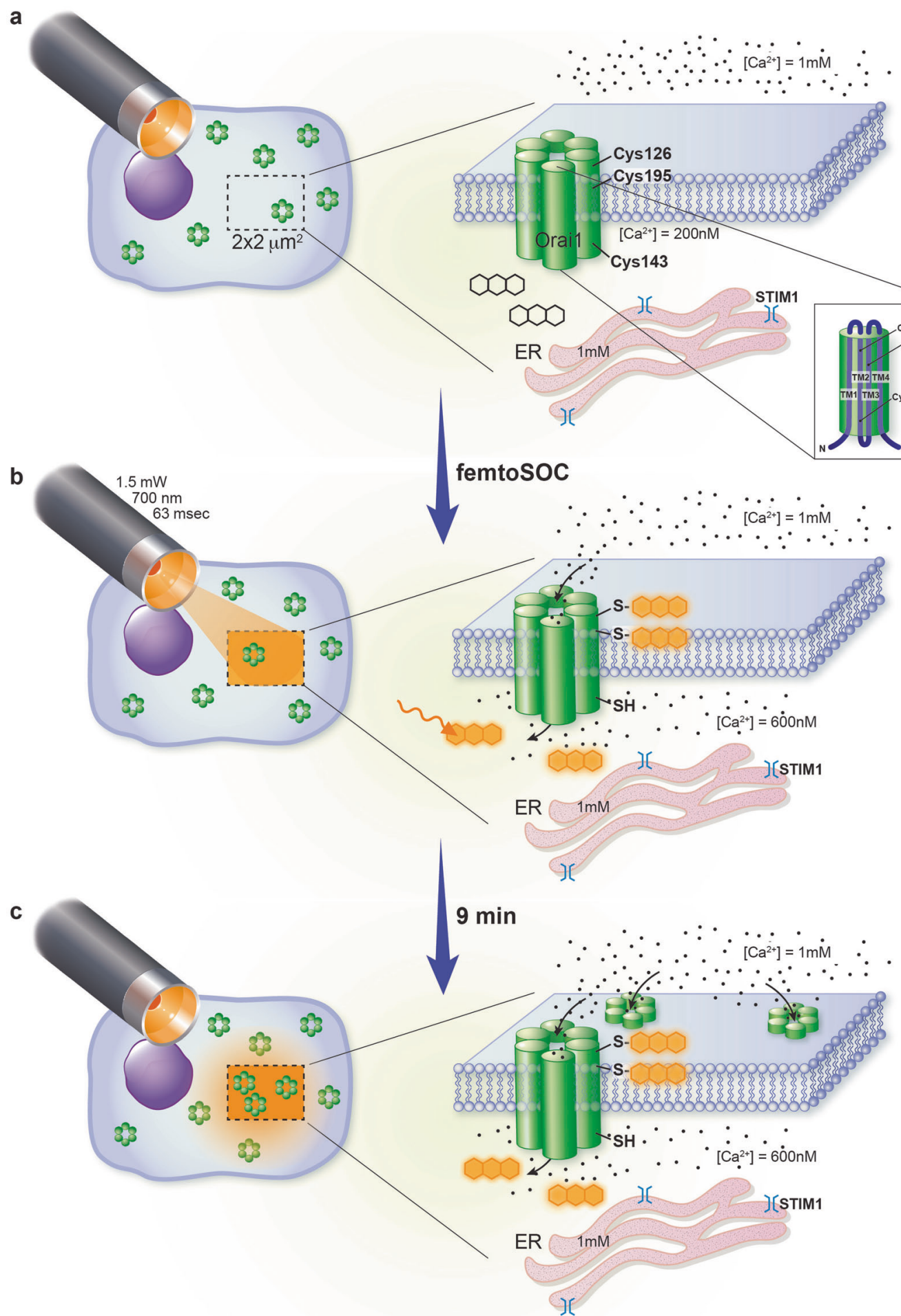

Fig. 1 Mechanism of Orai1 activation by femtoSOC. a In the resting state, Orai1 hexamers are dispersed throughout the PM, and STIM1 is located distantly within the ER membrane. Inset, the Orai1 channel topography showing the four transmembrane (TM) helices, and approximate location of active cysteine residues. b Femtosecond laser pulses from a multiphoton microscope $(1.5 \mathrm{~mW}, 700 \mathrm{~nm}, 63 \mathrm{~ms})$ are applied by scanning over a $2 \times 2 \mu \mathrm{m}^{2}$ area of the PM. Flavins in the region are photo-excited and bind to Cys 126 and Cys 195 residues in the Orai 1 molecule. This rapidly activates Orai1 channel opening, allowing extracellular $\mathrm{Ca}^{2+}$ to enter the cell. c After a further 9 min, Orai channels become aggregated and/or trapped within the femtoSOC-focused area. Throughout the activation, STIM proteins in the ER remain uninvolved. Extracellular and cytosolic Ca ${ }^{2+}$ concentrations are indicated by dots. The photo-activated flavin molecule is shown in orange. Illustration by Susan Weil-Kazzaz.

activation. Moreover, mutational analysis suggests that photoexcited flavins may form thiol-ether interactions with Cys 126 and Cys195 of Orai1. Thus, the C126S or C195S mutations prevent femtoSOC. These Cys residues reside within a compact area of intersection between the pore-forming TM1 and the TM2 and TM3 helices (Fig. 1), a region critical for channel gating. ${ }^{1}$ The $\mathrm{C} 126$ residue in TM2 is just 2 helical turns from the crucial $\mathrm{H} 134$ residue that appears to directly couple with and mediate opening of the 
pore itself. ${ }^{9}$ C195 in the TM3 helix is a major site for oxidative modification which induces hydrogen bonding with S239 in TM4, with powerful effects on STIM-mediated Orai1 activation. ${ }^{10}$

Overall, the studies of Cheng et al. ${ }^{7}$ reveal an unexpected and potentially very useful non-invasive means to activate $\mathrm{Ca}^{2+}$ signals in cells. The mechanism is intriguing although the reason for the specificity of femtoSOC on Orai1 channels is unknown. Nevertheless, the technique provides a simple, highly effective and non-damaging means to understand the intricate responses induced by spatially-directed $\mathrm{Ca}^{2+}$ signals.

\section{REFERENCES}

1. Zhou, Y. et al. PLoS Biol. 17, e3000413 (2019).

2. He, L. et al. Elife 4, e10024 (2015).

3. Kyung, T. et al. Nat. Biotechnol. 33, 1092-1096 (2015).

4. He, L. et al. Nat. Commun. 12, 164 (2021).

5. He, H., Kong, S. K. \& Chan, K. T. J. Biomed. Opt. 15, 057010 (2010).

6. Zhao, Y. et al. J. Biomed. Opt. 15, 035001 (2010).

7. Cheng, P. et al. Cell Res. https://doi.org/10.1038/s41422-020-00463-9 (2021).

8. Zhou, Y. et al. Proc. Natl. Acad. Sci. USA 115, E3398-E3407 (2018).

9. Yeung, P. S. et al. Proc. Natl. Acad. Sci. USA 115, E5193-E5202 (2018)

10. Niemeyer, B. A. Adv. Exp. Med. Biol. 993, 99-116 (2017). 